\title{
Knowledge of dentists, dental auxiliaries, and students regarding the COVID-19 pandemic in Saudi Arabia: a cross-sectional survey
}

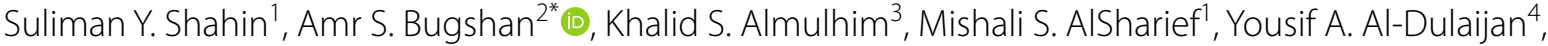 \\ Intisar Siddiqui ${ }^{5}$ and Faisal D. al-Qarni ${ }^{4}$
}

\begin{abstract}
Background: This study aimed to assess the knowledge of dental professionals in Saudi Arabia regarding severe acute respiratory syndrome coronavirus 2 (SARS-CoV-2) and coronavirus disease 2019 (COVID-19).

Methods: A questionnaire was developed to assess various dental professionals from both governmental and private sectors through online and social media outlets.

Results: A total of 1,033 questionnaires were collected (273 dental students, 193 dental auxiliary personnel, 544 dentists). In all, $63.4 \%$ of the respondents worked in hospitals. Of all the respondents, $44.9 \%, 33.4 \%$, and $21.7 \%$ worked in governmental clinics, academia, and the private sector, respectively. Overall knowledge of the incubation period and route of transmission of SARS-CoV-2 was consistent across all dental professions. Knowledge of hand-soap cleaning time was significantly different among dental professionals $(p<0.001)$. Dental professionals displayed significant disagreement on the survival of SARS-CoV-2 outside the host $(p<0.001)$. Furthermore, $75.1 \%$ of the respondents were reluctant to treat a suspected COVID-19 patient, and $92 \%$ of the participants believed that the mode of transmission was droplet inhalation. Fever, coughing, and shortness of breath were identified as the most common symptoms of COVID-19. Most standard methods of prevention in the dental office were selected by at least $50 \%$ of the participants.

Conclusions: Dental professionals seem to be consistent regarding their knowledge of the incubation period of SARS-CoV-2. However, knowledge of viral survivability and recommended hand-soap washing time was significantly variable among the professionals. A high degree of apprehension toward suspected COVID-19 patients existed among all dental professionals. Pandemic-awareness campaigns are essential among healthcare providers.
\end{abstract}

Keywords: Awareness, Dentists, Dental student, COVID-19, SARS-CoV-2

\section{Background}

On January 30, 2020, the World Health Organization (WHO) [1] announced a public health emergency of international significance regarding a global pneumonia epidemic. The WHO called this disease the novel viral pneumonia coronavirus disease 2019 (COVID-19)

\footnotetext{
*Correspondence: abugshan@iau.edu.sa

2 Department of Biomedical Dental Sciences, College of Dentistry, Imam

Abdulrahman Bin Faisal University, Dammam, Saudi Arabia

Full list of author information is available at the end of the article
}

on February 11, 2020. The first few cases of COVID-19 were identified in December 2019 in Wuhan, China, after genome sequencing of samples from patients presenting with pneumonia symptoms of unknown etiology [1]. The virus then spread to other provinces of China within weeks and became a global pandemic within a few months [2]. On March 2, 2020, Saudi Arabia announced its first case of severe acute respiratory syndrome coronavirus 2 (SARS-CoV-2) infection. Since then, the Saudi Ministry of Health has urged the general population to engage in social distancing and adopt sanitary habits and 
self-quarantine for a period of 14 days if an individual has recently been residing out of the kingdom. Coronaviruses are enveloped positive-strand RNA viruses with a crown-like appearance due to the presence of glycoprotein projections on their surfaces [3]. SARS-CoV-2 shares the same host receptor with SARS-CoV, namely, human angiotensin-converting enzyme 2 . The natural host of SARS-CoV-2 may be the bat, as it showed $96.2 \%$ wholegenome identity to BatCoV RaTG13 [4].

The common clinical symptoms of patients with novel viral pneumonia include fever, cough, myalgia, and exhaustion with an irregular chest computed tomography scan; less common symptoms are sputum development, headache, hemoptysis, and diarrhea $[5,6]$. The typical transmission routes of novel coronaviruses include direct (coughing, sneezing, and droplet inhalation) and contact (with oral, nasal, and eye mucous membranes) transmission [7].

A dental professional team is considered a high-risk group. Pathogenic microorganisms may be transmitted in dental settings through inhalation of airborne microorganisms that can stay trapped in the air for long periods [8]; direct contact with blood, oral fluids, or other patient materials [9]; contact of the conjunctival, nasal, or oral mucosa with droplets and microorganism-containing aerosols produced from an infected person and propelled by coughing and talking at a short distance without a mask $[10,11]$ or using high-pressure irrigation systems such as the handpiece or ultrasonic scalers; and indirect contact with contaminated instruments and/or environmental surfaces [12]. Therefore, it is crucial for dental professional teams to practice preventive measures against SARS-CoV-2 infection by focusing on hand hygiene, with an adequate hand-soap cleaning time of approximately $40 \mathrm{~s}$ [13], and personal protective equipment (PPE); ensuring an adequately ventilated space; and exercising caution when performing aerosol-generating procedures [11].

The aim of this study was to assess the knowledge of dentists and dental auxiliaries and students in Saudi Arabia regarding SARS-CoV-2, as they are considered a high-risk group for infection, and investigate the potential gaps in knowledge that may affect health and safety in the dental workplace.

\section{Methods}

A cross-sectional survey was conducted after ethical approval (EA\# 202049) was obtained from the Scientific Research Unit at the College of Dentistry, Imam Abdulrahman Bin Faisal University, Dammam, Saudi Arabia. The questionnaire was developed in English and further reviewed for face validation by two independent local reviewers. Major governmental hospitals, private clinics, and academic universities in various cities of all provinces of Saudi Arabia were approached. A corresponding link was sent to all targeted samples using different methods, including emails, text messages, and social media outlets. The targeted samples included dental students, dental interns, dental hygienists, dental assistants, general dentists, dental specialists, and consultants working in hospital and nonhospital governmental and private sectors. Respondents who were from outside Saudi Arabia and those who missed more than two questions were excluded from the study. To ensure the clarity and validity of the questions, a pilot test of the questionnaire was initially performed on a group of 100 dental professionals. The questionnaire was attached as Additional file 1.

The survey was constructed based on the latest literature resources about the characteristics and routes of transmission of SARS-CoV-2 and the signs and symptoms and prevention methods of COVID-19 [1-9, 13-16]. The first part of the questionnaire included the demographic information of the participants, that is, their age group, sex, geographic location of practice within Saudi Arabia, type of profession, primary work sector (academia, government, or private), and work setting (hospital or nonhospital). The second part of the questionnaire assessed the participants' awareness of the basic knowledge of SARS-CoV-2 and COVID-19. Dental professionals were asked about COVID-19 signs and symptoms and SARS-CoV-2 routes of transmission, incubation period, and viability outside the human body. The last part of the questionnaire included the methods of prevention and their attitude toward treating SARS-CoV2 -infected patients. All data were collected during March 2020.

SPSS-20.0 was used for statistical data analysis (IBM Corp. Released 2011. IBM SPSS Statistics for Macintosh, Version 20.0. Armonk, NY, USA: IBM Corp.). The results of all categorical variables are presented in terms of frequencies and percentages. The chi-square test was performed to compare the factors between work sectors, work settings, and professionals. All tests were performed at a significance level of $\alpha=0.05$.

\section{Results}

Out of 1033 questionnaires received, 20 were omitted because they were from outside Saudi Arabia. Some of the questionnaires were incomplete; those with more than one missing answer were excluded from the analysis.

\section{Reliability and validity}

A pilot sample size of 100 participants was selected for the reliability and validity of the research instrument. After standardizing all items, reasonably good interrater reliability was found (Cronbach's $\alpha=0.788$ ). Face 
validation of the questionnaire was performed by two independent local experts.

\section{Demographic results}

More than half of the participants were female, accounting for $53.5 \%(n=542)$ of the study sample, while men accounted for $46.5 \%(n=471)$ of the study sample. Half of the participants were 20 to $<30$ years old (50\%), 301 were 30 to $<40$ years old (29.7\%), 134 were 40 to $<50$ years old (13.2\%), and 71 were $\geq 50$ years old (7\%). Among the professions, the highest number of respondents were specialists/consultants $(\mathrm{n}=271,26.8 \%)$, followed by dental students $(\mathrm{n}=197,19.5 \%)$, general dentists $(\mathrm{n}=181$, $17.9 \%)$, dental assistants $(\mathrm{n}=156,15.4 \%)$, postgraduate residents $(\mathrm{n}=92,9.1 \%)$, dental interns $(\mathrm{n}=76,7.5 \%)$, and dental hygienists $(n=37,3.7 \%)$. Detailed distribution of participants' demographic characteristics is listed in Table 1.

\section{Awareness of SARS-CoV-2 and bivariate analysis results}

When evaluating the awareness of the survival period of SARS-CoV-2 outside a host, approximately half of the respondents $(\mathrm{n}=496,49.2 \%)$ believed that the survivability of the virus outside the host was a couple of hours, 360 (35.7\%) believed that the survivability of the virus was a couple of days, 71 (7\%) believed it was a couple of weeks, and $82(8.1 \%)$ did not know. In the between-group comparison, knowledge of the survivability of SARS-CoV-2 outside a host among those working in different settings and sectors was not significantly different $(p>0.05)$. However, when evaluating the various dental professions, a statistically significant difference was found among the various professions $(p<0.001)$. Among dental assistants, $17.9 \%(n=28)$ believed that the survivability of SARSCoV-2 was a couple of weeks, whereas only between $2.8 \%$ and $7.6 \%$ of the rest of the dental professionals believed the same concept. Furthermore, $48.3 \%(n=131)$ of specialists and consultants believed that the survivability of SARS-CoV-2 was a couple of days, while only $23.7 \%$ $(n=18), 25.6 \%(n=40)$, and $26.4 \%(n=52)$ of the interns, assistants, and students believed the same concept. This indicates a statistically significant disagreement among dental professionals regarding the survivability of SARSCoV-2 outside the host.

The awareness of the recommended hand-soap cleaning time to prevent SARS-CoV-2 infection was distributed as follows: 561 (55.6\%) responded $40 \mathrm{~s} ; 277$ (27.4\%), $20 \mathrm{~s} ; 155$ (15.3\%), $60 \mathrm{~s}$; and 17 (1.7\%) responded that they did not know the recommended hand-soap cleaning time for prevention. Again, in the between-group comparison, knowledge of the recommended hand-soap cleaning time to prevent SARS-CoV-2 infection among those working in different settings and sectors was not significantly
Table 1 Distribution of demographic characteristics of study participants

\begin{tabular}{lc}
\hline Variable & $\mathbf{n}(\%)$ \\
\hline Age (years) & \\
20 to $<30$ & $507(50 \%)$ \\
30 to $<40$ & $301(29.7 \%)$ \\
40 to $<50$ & $134(13.2 \%)$ \\
50 or above & $71(7 \%)$ \\
Profession & \\
Dental student & $197(19.5 \%)$ \\
Dental intern & $76(7.5 \%)$ \\
Dental assistant & $156(15.4 \%)$ \\
Dental hygienist & $37(3.7 \%)$ \\
Postgraduate resident & $92(9.1 \%)$ \\
General dentist & $181(17.9 \%)$ \\
Specialist/consultant & $271(26.8 \%)$ \\
Region & \\
Northern Region & $46(4.5 \%)$ \\
Southern Region & $107(10.6 \%)$ \\
Western Region & $226(22.3 \%)$ \\
Eastern Region & $367(36.2 \%)$ \\
Central Region & $267(26.4 \%)$ \\
Work sector & \\
Academia & $336(33.4 \%)$ \\
Government & $452(44.9 \%)$ \\
Private & $219(21.7 \%)$ \\
Work setting & \\
Hospital & $641(63.4 \%)$ \\
Nonhospital & $370(36.6 \%)$ \\
\hline &
\end{tabular}

different $(p>0.05)$. However, when evaluating the various dental professions, a statistically significant difference was found among the various professions with regard to the recommended hand-soap cleaning time $(p<0.001)$. The majority of dental professionals, except for dental assistants, responded in the range of $55.9 \%-69.4 \%$ on a recommended time of $40 \mathrm{~s}$, whereas only $25 \%(\mathrm{n}=39)$ of dental assistants answered the recommended $40 \mathrm{~s}$ of hand-soap cleaning time to prevent SARS-CoV-2 contamination.

Regarding the questions about willingness to treat a suspected COVID-19 patient, approximately threequarters of dental professionals $(\mathrm{n}=756,75.1 \%)$ did not want to treat a suspected COVID-19 patient. Bivariate analyses for between-group comparisons among those working in different settings and sectors and among the various dental professions regarding this question were not significantly different $(p>0.05)$. Table 2 and Table 3 illustrate the knowledge and awareness of SARS-CoV-2 and COVID-19 by work setting and sector and by dental 
Table 2 Pearson chi-square results for the knowledge and awareness of SARS-CoV-2 and COVID-19 by profession

\begin{tabular}{|c|c|c|c|c|c|c|c|c|c|}
\hline \multirow[t]{2}{*}{ Variable } & \multicolumn{8}{|c|}{ Dental profession } & \multirow[t]{2}{*}{$p$} \\
\hline & $\begin{array}{l}\text { Dental } \\
\text { student }\end{array}$ & Dental intern & $\begin{array}{l}\text { Dental } \\
\text { assistant }\end{array}$ & $\begin{array}{l}\text { Dental } \\
\text { hygienist }\end{array}$ & $\begin{array}{l}\text { Postgraduate } \\
\text { resident }\end{array}$ & $\begin{array}{l}\text { General } \\
\text { dentist }\end{array}$ & $\begin{array}{l}\text { Specialist } \\
\text { consultant }\end{array}$ & Total & \\
\hline \multicolumn{10}{|c|}{ Q1: Incubation period of SARS-CoV-2? } \\
\hline $1-7$ days & $3(1.5 \%)$ & $2(2.6 \%)$ & $4(2.6 \%)$ & $1(2.8 \%)$ & $4(4.4 \%)$ & $4(2.2 \%)$ & $9(3.3 \%)$ & 27 & \\
\hline $1-14$ days & $184(93.4 \%)$ & $71(93.4 \%)$ & $139(89.1 \%)$ & $35(97.2 \%)$ & $84(91.2 \%)$ & $165(91.7 \%)$ & $245(90.4 \%)$ & 923 & 0.866 \\
\hline $1-21$ days & $7(3.6 \%)$ & $3(4.0 \%)$ & $12(7.7 \%)$ & $0(0 \%)$ & $4(4.4 \%)$ & $10(5.6 \%)$ & $15(5.6 \%$ & 51 & \\
\hline Do not know & $3(1.5 \%)$ & $0(0 \%)$ & $1(0.6 \%)$ & $0(0 \%)$ & $0(0 \%)$ & $1(0.5 \%)$ & $2(0.7 \%)$ & 7 & \\
\hline Total & $197(100 \%)$ & $76(100 \%)$ & $156(100 \%)$ & $36(100 \%)$ & $92(100 \%)$ & $180(100 \%)$ & $271(100 \%)$ & 1008 & \\
\hline \multicolumn{10}{|c|}{ Q2: Survival of SARS-CoV-2 outside the host? } \\
\hline $\begin{array}{l}\text { Couple of } \\
\text { hours }\end{array}$ & $108(54.8 \%)$ & $48(63.2 \%)$ & 78 (50\%) & $22(61.1 \%)$ & $42(45.6 \%)$ & $83(46.1 \%)$ & $115(42.4 \%)$ & 496 & \\
\hline Couple of days & $52(26.4 \%)$ & 18 (23.7\%) & $40(25.6 \%)$ & 11 (30.5\%) & $39(42.4 \%)$ & 69 (38.3\%) & $131(48.3 \%)$ & 360 & $0.000 * *$ \\
\hline $\begin{array}{l}\text { Couple of } \\
\text { weeks }\end{array}$ & $10(5.1 \%)$ & $3(3.9 \%)$ & $28(18 \%)$ & $1(2.8 \%)$ & $7(7.6 \%)$ & $10(0.6 \%)$ & $12(4.5 \%)$ & 71 & \\
\hline Do not know & $27(13.7 \%)$ & 7 (9.2\%) & $10(6.4 \%)$ & $2(5.6 \%)$ & $4(4.4 \%)$ & $18(10 \%)$ & $13(4.8 \%)$ & 81 & \\
\hline Total & 197 (100\%) & $76(100 \%)$ & $156(100 \%)$ & $36(100 \%)$ & $92(100 \%)$ & 180 (100\%) & $271(100 \%)$ & 1008 & \\
\hline \multicolumn{10}{|c|}{ Q3: Recommended hand-soap cleaning time to prevent SARS-CoV-2-infection? } \\
\hline $\begin{array}{l}\text { Approximately } \\
20 \mathrm{~s}\end{array}$ & $35(17.8 \%)$ & $15(19.7 \%)$ & $60(38.5 \%)$ & $5(13.9 \%)$ & $19(20.7 \%)$ & $45(24.8 \%)$ & $98(36.3 \%)$ & 277 & \\
\hline $\begin{array}{l}\text { Approximately } \\
40 \mathrm{~s}\end{array}$ & $118(60.3 \%)$ & $44(57.9 \%)$ & $39(25.0 \%)$ & $25(69.4 \%)$ & $63(68.4 \%)$ & $121(66.9 \%)$ & $151(55.9 \%)$ & 561 & \\
\hline $\begin{array}{l}\text { Approximately } \\
60 \mathrm{~s}\end{array}$ & $38(19.3 \%)$ & $16(21.1 \%)$ & $56(35.9 \%)$ & $4(11.1 \%)$ & $6(6.5 \%)$ & $14(7.7 \%)$ & $18(6.7 \%)$ & 152 & $0.000^{* *}$ \\
\hline Do not know & $5(2.6 \%)$ & $1(1.3 \%)$ & $1(0.6 \%)$ & $2(5.6 \%)$ & $4(4.4 \%)$ & $1(0.6 \%)$ & $3(1.1 \%)$ & 17 & \\
\hline Total & $196(100 \%)$ & $76(100 \%)$ & $156(100 \%)$ & $36(100 \%)$ & $92(100 \%)$ & $181(100 \%)$ & $270(100 \%)$ & 1007 & \\
\hline \multicolumn{10}{|c|}{ Q4: Willingness to treat a suspected COVID-19 patient? } \\
\hline Yes & $52(26.4 \%)$ & $15(19.7 \%)$ & $44(28.8 \%)$ & $14(38.9 \%)$ & $16(17.4 \%)$ & $48(26.7 \%)$ & $62(23 \%)$ & 251 & \\
\hline No & $145(73.6 \%)$ & $61(80.3 \%)$ & $109(71.2 \%)$ & $22(61.1 \%)$ & $76(82.6 \%)$ & $132(73.3 \%)$ & $208(77 \%)$ & 753 & 0.129 \\
\hline Total & $197(100 \%)$ & $76(100 \%)$ & $153(100 \%)$ & $36(100 \%)$ & $92(100 \%)$ & $180(100 \%)$ & $270(100 \%)$ & 1004 & \\
\hline
\end{tabular}

All tests were performed at a significance level of $a=0.05$

* Significant at $p<0.05$

** Significant at $p<0.001$

profession. The Pearson chi-square results are shown as well.

The majority of the 1,013 participants $(n=932,92 \%)$ believed that the route of transmission was through droplet spread (sneezing or coughing). Approximately half of the participants $(\mathrm{n}=548$ dental professionals, 54.1\%) believed that the virus could be transmitted through direct contact with saliva, blood, or other body fluids. Moreover, only approximately one-third of the respondents believed that transmission could occur via direct skin-to-skin contact and airborne transmission, with $312(30.8 \%)$ and 305 (30.1\%) responses, respectively.

Almost all of the respondents believed fever to be a sign and symptom of COVID-19, with 1,000 responses (98.7\%). Furthermore, 944 (93.2\%) respondents believed coughing to be a sign and symptom. In addition, 940 (92.8\%) respondents believed shortness of breath was a common sign and symptom of the disease. The responses for the remaining COVID-19 signs and symptoms were as follows: 736 (72.7\%) responses for headache; 671 (66.2\%), shortness of breath; 492 (48.6\%), muscle pain; 323 (31.9\%), diarrhea; 295 (29.1\%), nasal congestion; 187 (18.5\%), vomiting; and 32 (3.2\%), skin rash.

The methods of SARS-CoV-2 transmission prevention in dental clinics among participants were believed to be as follows (from the highest response to the lowest): PPE (gloves, masks, and wrapping) with 973 (96.1\%) responses; hand-soap cleaning, 960 (94.8\%); clinic surface disinfection, $877(86.6 \%)$; hand sanitizers, 873 (86.2\%); rubber dam isolation, 620 (61.2\%); utilization of an isolated clinic, 592 (58.4\%); adequate ventilation, 579 (57.2\%); use of preoperative chlorhexidine mouthwash, 360 (35.5\%); and use of preoperative hydrogen peroxide mouthwash, 207 (20.4\%). Table 4 summarizes the responses of all dental professionals with regard to awareness of the route of SARS-CoV-2 transmission, signs and 
Table 3 Pearson chi-square results for the knowledge and awareness of SARS-CoV-2 and COVID-19 by work settings and sectors

\begin{tabular}{|c|c|c|c|c|c|c|c|c|c|}
\hline \multirow[t]{2}{*}{ Variable } & \multicolumn{3}{|c|}{ Work setting } & \multirow[t]{2}{*}{$p$} & \multicolumn{4}{|l|}{ Work sector } & \multirow[t]{2}{*}{$p$} \\
\hline & Hospital & Nonhospital & Total & & Academia & Government & Private & Total & \\
\hline \multicolumn{10}{|c|}{ Q1: Incubation period of SARS-CoV-2? } \\
\hline $1-7$ days & $18(2.8 \%)$ & $9(2.4 \%)$ & 27 & & $8(2.4 \%)$ & $10(2.2 \%)$ & $9(4.1 \%)$ & 27 & \\
\hline $1-14$ days & $582(91.0 \%)$ & $342(92.7 \%)$ & 924 & 0.587 & $309(92.0 \%)$ & $418(92.7 \%)$ & $193(88.5 \%)$ & 920 & 0.648 \\
\hline $1-21$ days & $34(5.3 \%)$ & $17(4.6 \%)$ & 51 & & $16(4.7 \%)$ & $21(4.7 \%)$ & $14(6.4 \%)$ & 51 & \\
\hline Do not know & $6(0.9 \%)$ & $1(0.3 \%)$ & 7 & & $3(0.9 \%)$ & $2(0.4 \%)$ & $2(1.0 \%)$ & 7 & \\
\hline Total & $640(100 \%)$ & $369(100 \%)$ & 1009 & & $336(100 \%)$ & $451(100 \%)$ & $218(100 \%)$ & 1005 & \\
\hline \multicolumn{10}{|c|}{ Q2: Survival of SARS-CoV-2 outside the host? } \\
\hline Couple of hours & $323(50.5 \%)$ & $173(46.9 \%)$ & 496 & 0.146 & $163(48.5 \%)$ & $222(49.3 \%)$ & $111(50.7 \%)$ & 496 & \\
\hline Couple of days & $213(33.3 \%)$ & $147(39.8 \%)$ & 360 & & $133(39.6 \%)$ & $157(34.9 \%)$ & $67(30.6 \%)$ & 357 & 0.083 \\
\hline Couple of weeks & $46(7.2 \%)$ & $25(6.8 \%)$ & 71 & & $15(4.5 \%)$ & $39(8.7 \%)$ & $17(7.8 \%)$ & 71 & \\
\hline Do not know & $58(9.0 \%)$ & $24(6.5 \%)$ & 82 & & $25(7.4 \%)$ & $32(7.1 \%)$ & $24(10.9 \%)$ & 81 & \\
\hline Total & $640(100 \%)$ & $369(100 \%)$ & 1009 & & $336(100 \%)$ & $450(100 \%)$ & $219(100 \%)$ & 1005 & \\
\hline \multicolumn{10}{|c|}{ Q3: Recommended hand-soap cleaning time to prevent SARS-CoV-2 infection? } \\
\hline Approximately $20 \mathrm{~s}$ & $163(25.5 \%)$ & $113(30.5 \%)$ & 276 & & $95(28.4 \%)$ & $124(27.5 \%)$ & $56(25.6 \%)$ & 275 & \\
\hline Approximately $40 \mathrm{~s}$ & $359(56.2 \%)$ & $201(54.3 \%)$ & 560 & 0.244 & $179(53.6 \%)$ & $251(55.7 \%)$ & $128(58.4 \%)$ & 558 & 0.060 \\
\hline Approximately $60 \mathrm{~s}$ & $106(16.6 \%)$ & $49(13.2 \%)$ & 155 & & $48(14.4 \%)$ & $73(16.2 \%)$ & $33(15.1 \%)$ & 154 & \\
\hline Do not know & $10(2.7 \%)$ & $7(2.0 \%)$ & 17 & & $12(3.6 \%)$ & $3(0.6 \%)$ & $2(0.9 \%)$ & 17 & \\
\hline Total & $638(100 \%)$ & $370(100 \%)$ & 1008 & & $334(100 \%)$ & $451(100 \%)$ & $219(100 \%)$ & 1004 & \\
\hline \multicolumn{10}{|c|}{ Q4: Willingness to treat a suspected COVID-19 patient? } \\
\hline Yes & $164(25.7 \%)$ & $87(23.7 \%)$ & 251 & 0.489 & $90(26.9 \%)$ & $113(25.1 \%)$ & $47(21.6 \%)$ & 250 & \\
\hline No & $475(74.3 \%)$ & $280(76.3 \%)$ & 755 & & $244(73.1 \%)$ & $337(74.9 \%)$ & $171(78.4 \%)$ & 752 & 0.358 \\
\hline Total & $639(100 \%)$ & $367(100 \%)$ & 1006 & & $334(100 \%)$ & $450(100 \%)$ & $218(100 \%)$ & 1002 & \\
\hline
\end{tabular}

symptoms of COVID-19, and methods of transmission prevention in dental clinics.

Logistic regression analysis was performed by taking the willingness of the dentist to treat suspected COVID19 patients as an independent binary variable and sex, age, practice status, work sector, and dental service set up as independent covariates to evaluate the effect of these covariates on the outcome variable related to the perception of dental professionals regarding treating suspected COVID-19 patients.

The analysis revealed 2 predictors of willingness to treat suspected COVID-19 patients, i.e., dental assistants $(p=0.047)$ and dental hygienists $(p=0.012)$. Postgraduate residents were the least willing to treat COVID19-suspected patients, whereas dental assistants were 2 times $(\mathrm{OR}=1.92)$ and dental hygienists were 3 times $(\mathrm{OR}=3.02)$ more likely willing than postgraduate residents to treat COVID-19-suspected patients. There was a nonsignificant association of willingness of dentists to treat COVID-19 patients with regards to sex $(\mathrm{OR}=1.12)$, younger versus older age $(\mathrm{OR}=1.07)$, academic sector $(\mathrm{OR}=1.34)$ and government sector $(\mathrm{OR}=1.22)$ versus private sector, and hospital versus nonhospital setup $(\mathrm{OR}=1.11)$. Table 5 highlights the odds ratio of willingness to treat suspected COVID-19 patients by various variables.

\section{Discussion}

Due to its high mortality rate, SARS-CoV-2 and the associated debilitating COVID-19 are emerging topics that have recently received great attention and have undergone intense investigation. This pandemic disease is highly infectious, as the number of infected patients exceeded 2 million worldwide in $<6$ months, as reported by the WHO [14].

This survey assessed the degree of awareness of dental professionals, as they are typically in close contact with patients when delivering oral healthcare services.

Based on our results, the overall knowledge about the incubation period, route of transmission, and recommended hand-soap cleaning time to prevent SARSCoV-2 was consistent across all dental professions, except for dental assistants, where only $25 \%$ of them believed the recommended hand-soap cleaning time was $40 \mathrm{~s}$. This clearly emphasizes the significance of the adequacy of viral prevention knowledge across all dental professionals and auxiliaries during pandemic periods. Overall, these results show an improvement in 
Table 4 A summary of the awareness of the route of SARS-CoV-2 transmission, signs and symptoms of COVID-19, and methods of SARS-CoV-2 transmission prevention in dental clinics

\begin{tabular}{|c|c|c|}
\hline & Frequency & Percent \\
\hline \multicolumn{3}{|l|}{ Q5: Route of SARS-CoV-2 transmission } \\
\hline Direct skin-to-skin transmission & 312 & 30.8 \\
\hline Direct fluid transmission (saliva, body fluids, or blood) & 548 & 54.1 \\
\hline Droplet spread (sneezing or coughing) & 932 & 92 \\
\hline Airborne transmission (air or dust) & 305 & 30.1 \\
\hline \multicolumn{3}{|l|}{ Q6: Signs and symptoms of COVID-19 } \\
\hline Fever & 1000 & 98.7 \\
\hline Headache & 736 & 72.7 \\
\hline Diarrhea & 323 & 31.9 \\
\hline Vomiting & 187 & 18.5 \\
\hline Muscle pain & 492 & 48.6 \\
\hline Coughing & 944 & 93.2 \\
\hline Shortness of breath & 940 & 92.8 \\
\hline Sore throat & 671 & 66.2 \\
\hline Nasal congestion & 295 & 29.1 \\
\hline Skin rash & 32 & 3.2 \\
\hline \multicolumn{3}{|l|}{ Q7: Methods of SARS-CoV-2 transmission prevention in dental clinics } \\
\hline Hand-soap cleaning & 960 & 94.8 \\
\hline Hand sanitizers & 873 & 86.2 \\
\hline Personal protective equipment (gloves, masks, and wrapping) & 973 & 96.1 \\
\hline Preoperative chlorhexidine mouthwash & 360 & 35.5 \\
\hline Preoperative hydrogen peroxide mouthwash & 207 & 20.4 \\
\hline Rubber dam isolation & 620 & 61.2 \\
\hline Clinic surface disinfection & 877 & 86.6 \\
\hline Adequate ventilation & 579 & 57.2 \\
\hline Utilization of an isolated clinic & 592 & 58.4 \\
\hline
\end{tabular}

knowledge compared with the results found in a previous survey of dentists in Saudi Arabia about SARS$\mathrm{CoV}$ [17]. Although modestly answered among all professionals, the highest response was among dental specialists/consultants, with only $48.3 \%$ believing that the survivability of SARS-CoV-2 outside the host was a couple of days. There seemed to be a significant disagreement among the dental professionals regarding the question of SARS-CoV-2 survivability $(p<0.001)$. Regarding general knowledge about SARS-CoV-2, our study showed a relatively lower percentage of individuals who answered "I do not know" to questions regarding the incubation period and recommended hand-soap cleaning time $(0.69 \%$ and $1.69 \%$, respectively) compared with viral survival outside the host (10.04\%). This may be because local health authorities have utilized all media outlets toward a community-wide awareness campaign covering the pandemic, which had hand-soap cleaning time being one of the most shared preventive methods promoted. In contrast, the survival of the virus outside the host may be confusing due to various survival times related to different surfaces $[8,18]$. This further emphasizes the significance of knowledge on viral survival to ensure adequate preventive measures in the working environment while treating suspected COVID-19 patients.

The majority of respondents, regardless of professional category, were reluctant to treat a patient under suspicion of having COVID-19, which indicated a certain level of apprehension. This is thought to be due to the general guidelines conveyed to caregivers by local authorities, that is, to limit dental procedures to urgent or emergency cases only and refrain from elective procedures. Regarding willingness to treat suspected patients with COVID-19, dental hygienists (38.89\%) were most willing to treat suspected patients. In contrast, compared with the remaining groups, postgraduate dental residents $(17.39 \%)$ were least willing. This is an interesting outcome, as dental hygiene is considered to have mostly elective procedures compared with general or specialized dentistry. Future studies addressing the level of apprehension among dental professionals are warranted. 
Table 5 Predictors of willingness of dentist to treat suspected Covid-19 patient

\begin{tabular}{|c|c|c|c|c|}
\hline \multirow[t]{2}{*}{ Factors } & \multicolumn{2}{|c|}{$\begin{array}{l}\text { Willingness } \\
\text { of dentist }\end{array}$} & \multirow{2}{*}{$\begin{array}{l}\text { Odd ratio } \\
\text { OR }(95 \% \mathrm{Cl})\end{array}$} & \multirow{2}{*}{$\begin{array}{l}p \text { value } \\
\text { Sig }\end{array}$} \\
\hline & $\begin{array}{l}\text { Yes } \\
(n=251)\end{array}$ & $\begin{array}{l}\text { No } \\
(n=756)\end{array}$ & & \\
\hline Gender (Female) & $129(51.4)$ & $409(54.1)$ & $1.12(0.84-1.48)$ & 0.456 \\
\hline Age (20 to < 30 years) & $130(51.8)$ & $373(49.3)$ & $1.07(0.73-1.55)$ & 0.738 \\
\hline Age (30 to < 40 years) & $71(28.3)$ & $230(30.4)$ & $0.94(0.62-1.43)$ & 0.788 \\
\hline Age (40 to < 50 years) & $34(13.5)$ & $98(13.0)$ & $1.19(0.60-2.35)$ & 0.612 \\
\hline Age (> 50 years) & $16(6.4)$ & $55(7.3)$ & - & - \\
\hline Dental student & $52(20.7)$ & $145(19.3)$ & $1.70(0.91-3.18)$ & 0.095 \\
\hline Dental intern & $15(6.0)$ & $61(8.1)$ & $1.17(0.53-2.55)$ & 0.697 \\
\hline Dental assistant & $44(17.5)^{*}$ & $109(14.5)$ & $1.92(1.01-3.65)$ & $0.047^{*}$ \\
\hline Dental hygienist & $14(17.5)^{*}$ & $22(2.9)$ & $3.02(1.28-7.14)$ & $0.012^{*}$ \\
\hline General dentist & $48(19.1)$ & $132(17.5)$ & $1.73(0.92-3.25)$ & 0.090 \\
\hline Specialist/consultant & $62(24.7)$ & $208(27.6)$ & $1.42(0.77-2.60)$ & 0.263 \\
\hline Postgraduate resident & $16(6.4)$ & $76(10.1)$ & - & - \\
\hline Academic sector & $90(36.0)$ & $244(32.4)$ & $1.34(0.90-2.01)$ & 0.153 \\
\hline Government sector & $113(45.2)$ & $337(44.8)$ & $1.22(0.83-1.80)$ & 0.314 \\
\hline Hospital setup & $164(65.3)$ & $475(62.9)$ & $1.11(0.82-1.50)$ & 0.489 \\
\hline
\end{tabular}

*Indicates statistical significance

Regarding the question about modes of transmission, the majority of the participants (92\%) believed that the mode of transmission was droplet infection, followed by direct fluid transmission (54.1\%) and direct skin-toskin contact (30.8\%). However, $30.1 \%$ of the participants also believed that the virus was airborne transmissible, which is clearly a misconception, as the disease is transmitted by aerosols [3]. The present study showed that the majority of the participants identified fever, coughing, and shortness of breath as the most common symptoms related to COVID-19 (98.7\%, 93.2\%, and 92.8\%, respectively), which indicated a high degree of knowledge pertinent to this area. This is an intuitive finding considering that fever screenings have been increasingly prevalent at establishment entrances throughout the pandemic. Furthermore, previous epidemics of SARS-CoV and Middle East respiratory syndrome-related coronavirus (MERS$\mathrm{CoV})$ may explain the degree of knowledge regarding common respiratory symptoms of coronaviruses.

To prevent SARS-CoV-2 infection in dental clinics, dental professionals should follow standard precautions, which include appropriate hand hygiene, personal protective barriers (gloves, masks, face shields), use of preoperative mouthwash ( $1 \%$ hydrogen peroxide), rubber dam isolation, and implementation of effective and strict disinfection measures [15]. It is worth highlighting that $54.6 \%$ and $51.6 \%$ of dental assistants and hygienists, respectively, believed that the use of preoperative chlorhexidine mouthwash (0.12\%), which is antibacterial, was a preventative measure against SARS-CoV-2. On the other hand, $84.9 \%$ and 93.5\% of dental assistants and hygienists, respectively, did not believe that rubber dam isolation was a significant measure of SARS-CoV-2 prevention. This implies the presence of a gap in knowledge regarding preventive measures among the dental team.

Interestingly, dental assistants $(p=0.047)$ and dental hygienists $(p=0.012)$ had higher odds of willingness to treat suspected COVID-19 patients than postgraduate residents, who had the lowest willingness to treat these patients. This could be explained by less experience in dental practice in regard to residents in treating patients with medical conditions.

The representative sample of 1013 dental professionals covering all regions of Saudi Arabia is a strength of this study, as it is consistent with the distribution of the population within each region [16]. Furthermore, a thorough distribution among all dental professionals and dental work settings was achieved to act as a realistic representation of the country's dental work force. To the best of our knowledge, this is the first representative survey to provide insight into the knowledge and perception of SARS-CoV-2 by dentists and dental auxiliaries and students in Saudi Arabia. Furthermore, the distribution of male and female participants was balanced (46.5\% and 53.5\%, respectively). However, this study has several limitations. In addition to the general limitations of a cross-sectional study and online surveys, one of our limitations was the dichotomous nature of the question as to whether the practitioner was willing to treat a SARS-CoV-2 patient. Information pertaining to participants who answered "No" but who would otherwise be willing to treat emergency patients only may have been lost, as the options were only "Yes" or "No," especially with a considerable number of dental procedures being elective or at least of a nonurgent nature. In addition, with the high volume of information influx from media outlets in combination with a higher than average viewership due to curfews and lockdowns, the status quo of general perceptions may shift on a weekly or even daily basis, rendering this survey limited to a specific snapshot of time. A further limitation was the variation in the number of responses among the various dental professions. In addition, factors such as length of practice, nationality, and race were not considered in this study. Detailed investigation on practice in dental clinics in regard to the COVID-19 pandemic is warranted. Further studies addressing the abovementioned limitations are needed. This study can be used as a benchmark for general 
knowledge and attitudes toward pandemics in developing countries with an established dental healthcare system.

\section{Conclusion}

The knowledge among dental professionals on SARSCoV-2 and COVID-19 seems to be consistent when evaluating the viral incubation period. However, viral survivability and recommended hand-soap washing time were significantly variable among the professionals. Furthermore, a certain level of apprehension toward suspected COVID-19 patients existed among all dental professionals. Dental assistants and hygienists had higher odds of willingness to treat suspected COVID19 patients. During a novel pandemic, a joint effort to strengthen knowledge among dentists and dental auxiliaries needs to be reinforced within the dental professional team. Studies comparing knowledge at every stage of the pandemic are recommended.

\section{Supplementary Information}

The online version contains supplementary material available at https://doi. org/10.1186/s12903-020-01361-7.

Additional file 1. The questionnaire distributed to the respondents.

\section{Abbreviations}

SARS-CoV-2: Severe acute respiratory syndrome coronavirus 2; COVID-19: Coronavirus disease 2019; WHO: World Health Organization; PPE: Personal protective equipment.

\section{Acknowledgements}

Not applicable.

\section{Authors' contributions}

Conceptualization: S.H. and A.B. Methodology: S.H., A.B., K.A. and F.Q. Software: S.H. Validation: S.H. and M.A. Formal analysis: S.H. and I.S. Statistical analysis: I.S. Writing —original draft preparation: A.B., K.A., M.A., Y.D. and F.Q. Writingreview and editing: K.A., M.A., Y.D. and F.Q. All authors have read and agreed to the published version of the manuscript. All authors read and approved the final manuscript

\section{Funding}

This research received no funding.

\section{Availability of data and materials}

The datasets generated and analyzed during the current research are not publicly available, but they are available from the corresponding author on reasonable request.

\section{Ethics approval and consent to participate}

Online written consent was obtained before completing the survey and respondents were informed that their participation in the study was entirely voluntary. The Scientific Research Unit at the College of Dentistry, Imam Abdulrahman Bin Faisal University approved this study and the method of obtaining the consent (EA\# 202049). Respondents were assured of the confidentiality of the information provided and were informed that they could withdraw from the study at any time or opt not to answer any of the questions in the survey.

\section{Consent for publication}

Not applicable.

\section{Competing interests}

The authors declare that they have no competing interests.

\section{Author details}

${ }^{1}$ Department of Preventive Dental Sciences, College of Dentistry, Imam Abdulrahman Bin Faisal University, Dammam, Saudi Arabia. ${ }^{2}$ Department of Biomedical Dental Sciences, College of Dentistry, Imam Abdulrahman Bin Faisal University, Dammam, Saudi Arabia. ${ }^{3}$ Department of Restorative Dental Sciences, College of Dentistry, Imam Abdulrahman Bin Faisal University, Dammam, Saudi Arabia. ${ }^{4}$ Department of Substitutive Dental Sciences, College of Dentistry, Imam Abdulrahman Bin Faisal University, Dammam, Saudi Arabia. ${ }^{5}$ Vice Deanship for Studies, Development and Community Service, College of Dentistry, Imam Abdulrahman Bin Faisal University, Dammam, Saudi Arabia.

Received: 4 August 2020 Accepted: 10 December 2020

Published online: 21 December 2020

\section{References}

1. Zhu N, et al. A novel coronavirus from patients with pneumonia in China, 2019. N Engl J Med. 2020:382:727-33.

2. She J, et al. 2019 novel coronavirus of pneumonia in Wuhan, China: emerging attack and management strategies. Clin Transl Med. 2020;9:19.

3. Cascella M, et al. Features, evaluation and treatment coronavirus (COVID19). In StatPearls. 2020: Treasure Island (FL).

4. Zhou P, et al. A pneumonia outbreak associated with a new coronavirus of probable bat origin. Nature. 2020;579:270-3.

5. Huang C, et al. Clinical features of patients infected with 2019 novel coronavirus in Wuhan, China. Lancet. 2020;395:497-506.

6. Wang D, et al. Clinical characteristics of 138 hospitalized patients with 2019 novel coronavirus-infected pneumonia in Wuhan, China. JAMA 2020

7. Lu CW, Liu XF, Jia ZF. 2019-nCoV transmission through the ocular surface must not be ignored. Lancet. 2020:395:e39.

8. Kampf $\mathrm{G}$, et al. Persistence of coronaviruses on inanimate surfaces and their inactivation with biocidal agents. J Hosp Infect. 2020;104:246-51.

9. Chen J. Pathogenicity and transmissibility of 2019-nCoV-A quick overview and comparison with other emerging viruses. Microbes Infect. 2020;22:69-71.

10. Cleveland JL, et al. Transmission of blood-borne pathogens in US dental health care settings: 2016 update. J Am Dent Assoc. 2016;147:729-38.

11. Harrel SK, Molinari J. Aerosols and splatter in dentistry: a brief review of the literature and infection control implications. J Am Dent Assoc. 2004;135:429-37.

12. Liu L, et al. Epithelial cells lining salivary gland ducts are early target cells of severe acute respiratory syndrome coronavirus infection in the upper respiratory tracts of rhesus macaques. J Virol. 2011;85:4025-30.

13. Saudi Ministry of Health (MOH) COVID-19 Awareness Guidelines. Available from: https://www.moh.gov.sa/awarenessplateform/VariousTopics/ Documents/PreventCOVID19-Eng.pdf. Accessed on 20 April 2020.

14. WHO Coronavirus disease 2019 (COVID-19) Situation Report-88. Available from: https://www.who.int/docs/default-source/coronaviruse/situa tion-reports/20200417-sitrep-88-covid-191 b6cccd94f8b4f219377bff 55719a6ed.pdf?sfvrsn=ebe78315_6. Accessed on 20 April 2020.

15. Gaffar BO, et al. Knowledge and practices of dentists regarding MERS-CoV: a cross-sectional survey in Saudi Arabia. Saudi Med J. 2019;40:714-20.

16. Otter JA, et al. Transmission of SARS and MERS coronaviruses and influenza virus in healthcare settings: the possible role of dry surface contamination. J Hosp Infect. 2016;92:235-50.

17. Peng $X$, et al. Transmission routes of 2019-nCoV and controls in dental practice. Int J Oral Sci. 2020;12:9.

18. Saudi Census. Available from: https://saudicensus.sa/. Accessed on 20 April 2020.

\section{Publisher's Note}

Springer Nature remains neutral with regard to jurisdictional claims in published maps and institutional affiliations. 